\title{
LENGTH BIASED BETA-PARETO DISTRIBUTION AND ITS STRUCTURAL PROPERTIES WITH APPLICATION
}

\author{
Nareerat Nanuwong and Winai Bodhisuwan \\ Department of Statistics, Faculty of Science, Kasetsart University, Bangkok, 10900, Thailand
}

Received 2013-11-20; Revised 2013-11-26; Accepted 2014-01-11

\begin{abstract}
The concept of length biased distribution can be employed in development of proper models for lifetime data. Its method is adjusting the original probability density function from real data and the expectation of those data. This adjustment can bring about to correct conclusions of models. Therefore, we introduced the Length Biased Beta-Pareto (LBBP) distribution, so-called a new generalized of Pareto distribution in this article. The distribution is more flexible and has some interesting properties such as hazard rate, Renyi and Shannon entropies and other types of distribution. There are several sub-models include in the length biased Pareto, arcsine, log-beta, exponential and beta-Pareto distributions. We apply maximum likelihood estimation to estimate parameters of the distribution. We illustrate the superiority of the LBBP distribution to Norwegian fire claims data. The LBBP distribution seems to be the most appropriate model for this data set, since it provides a significantly better fit than the length biased Pareto and the beta-Pareto distributions. We hope that the LBBP distribution is an alternative distribution can be used in lifetime data analysis and other fields.
\end{abstract}

Keywords: Length Biased Beta-Pareto Distribution, Hazard Rate, Renyi and Shannon Entropies, Maximum Likelihood Estimation, Lifetime Data

\section{INTRODUCTION}

The problem of determining proper model for interested information are one thing that important for data analysts. One major benefit of the weighted distribution theory provides a unifying approach for these problems was proposed by Rao (1965). It is ability of fitting skewed data that will not be properly fitted by existing distributions. Many authors including the concept of weighted distribution for example; Patil and Rao (1978) examine some general models leading to weighted distributions with weight functions not necessarily bounded by unity and studied length biased (size biased) sampling with applications to wildlife populations and human families. Characteristics of many length biased distributions, preservation stability results and comparisons for weighted and length biased distributions were presented; (Khattree, 1989; Oluyede and George, 2002).
Probability weighted moment inequalities and variability orderings for weighted and unweighted reliability measures and related functions were presented by Oluyede (2006). Also, stochastic comparisons and moment inequalities were given. More recently, Das and Roy (2011) developed the length biased form of the weighted Weibull distribution and discussed various properties of it. The result of this distribution suggested a good fitted to consecutive years data. Further, Ahmed et al. (2013) presented the class of size biased generalized gamma distribution and derived Shannon entropy which is the measure of the uncertainty in this distribution. Alkarni (2012) introduced lifetime class with decreasing failure rate by compounding truncated logarithmic distribution with any proper continuous lifetime distribution.

Statistical models and methods for lifetime data analysis are extensively used in many fields, including the biomedical sciences, engineering and management; Corresponding Author: Winai Bodhisuwan, Department of Statistics, Faculty of Science, Kasetsart University, P.O. Box 1086, Bangkok, 10900, Thailand Tel: +662 5625444 Ext. 3876 Fax: +662 9428384 
(Lawless, 2011). The Pareto distribution is broadly used to modelling a diverse range of lifetime observable phenomena. Many transformations and generalization of the Pareto distribution have been proposed in order to get more flexible models; (Newman, 2005; Ali and Nadarajah, 2006; Manas, 2011; Nassar and Nada, 2013). Moreover, Johnson et al. (1995) and Nadarajah (2005) discussed other types of Pareto distribution more than the Probability Density Function (PDF) of Pareto density. Different methods may be used to introduce a shape parameter to the Pareto model.

Recently, The Beta-Pareto (BP) distribution was introduced by Akinsete et al. (2008). It is a continuous distribution that is found to be unimodal and reduces to some existing distributions that are known in the literature. A random variable (r.v.) $\mathrm{X}$ has a $\mathrm{BP}$ distribution if its PDF with parameters $\alpha, \beta, \theta$ and $\gamma$ as follows Equation (1):

$$
f(x)=\frac{\gamma}{\theta B(\alpha, \beta)}\left[1-\left(\frac{x}{\theta}\right)^{-\gamma}\right]^{\alpha-1}\left(\frac{x}{\theta}\right)^{-\gamma \beta-1}, x \geq \theta ; \alpha, \beta, \theta, \gamma>0
$$

Where:

$$
\mathrm{B}(\alpha, \beta)=\frac{\Gamma(\alpha) \Gamma(\beta)}{\Gamma(\alpha+\beta)}
$$

It is easy to prove the mean of PDF in (1) follows form Equation (2):

$$
\mathrm{E}(\mathrm{X})=\frac{\theta \Gamma(\alpha+\beta) \Gamma(\beta-1 / \gamma)}{\Gamma(\beta) \Gamma(\alpha+\beta-1 / \gamma)}
$$

The rth moments of $\mathrm{X}$ is given by Equation (3):

$$
E\left(X^{r}\right)=\theta^{r} \frac{B(\alpha, \beta-r / \gamma)}{B(\alpha, \beta)}, r=1,2,3, \ldots
$$

The BP distribution can be applied to some phenomena events flood data sets, it provides a significantly better fit than the Pareto, Weibull and generalized Pareto distributions.

In this work, we propose a new distribution which is called the Length Biased Beta-Pareto (LBBP) distribution. The article is outlined as follows: In Section 2, we introduce the LBBP distribution and illustrate some sub-models of distribution and provide plots of the density function. We investigate some mathematical properties are the hazard rate of distribution and devoted to the discussion on the rth moments. Rényi and Shannon entropies are discussed in this section. Maximum Likelihood Estimation (MLE) is addressed in Section 3 and we provide application of the LBBP distribution to real data set in this section and discussion in Section 4. Finally, we offer some concluding remarks on the main results and their significance.

\section{MATERIALS AND METHODS}

\subsection{Length Biased Beta-Pareto Distribution}

This section, we derived the shape of the PDF for the LBBP distribution and consider its some sub-models.

\section{Definition 1:}

Let $X$ be a nonnegative r.v. with $\operatorname{PDF} f(x)$ and assuming that $\mathrm{E}(\mathrm{X})<\infty$. Then the $\mathrm{PDF} \mathrm{f}_{\mathrm{L}}(\mathrm{x})$ for a length biased distribution of $\mathrm{X}$ can be obtained by:

$$
f_{L}(x)=\frac{x f(x)}{E(X)}
$$

\section{Theorem 1:}

Let $\mathrm{X} \sim \operatorname{LBBP}(\alpha, \beta, \theta, \gamma)$. The PDF and Cumulative Distribution Function (CDF) of $\mathrm{X}$ are given respectively as Equation (4):

$$
\begin{aligned}
& \mathrm{f}_{\mathrm{L}}(\mathrm{x})=\frac{\gamma}{\theta \mathrm{B}(\alpha, \beta-1 / \gamma)}\left[1-\left(\frac{\mathrm{x}}{\theta}\right)^{-\gamma}\right]^{\alpha-1}\left(\frac{\mathrm{x}}{\theta}\right)^{-\gamma \beta}, \\
& \mathrm{x} \geq \theta ; \alpha, \beta, \theta, \gamma>0
\end{aligned}
$$

And Equation (5):

$$
F_{L}(x)=1-\frac{B(z ; \beta-1 / \gamma, \alpha)}{B(\alpha, \beta-1 / \gamma)}, z=(x / \theta)^{-\gamma}
$$

\section{Proof:}

Substitute (1) and (2) in Definition 1, then PDF for LBBP r.v. can be obtained:

$$
\begin{aligned}
\mathrm{f}_{\mathrm{L}}(\mathrm{x}) & =\frac{\frac{\gamma \mathrm{x}}{\theta \mathrm{B}(\alpha, \beta)}\left[1-\left(\frac{\mathrm{x}}{\theta}\right)^{-\gamma}\right]^{\alpha-1}\left(\frac{\mathrm{x}}{\theta}\right)^{-\gamma \beta-1}}{\frac{\theta \Gamma(\alpha+\beta) \Gamma(\beta-1 / \gamma)}{\Gamma(\beta) \Gamma(\alpha+\beta-1 / \gamma)}} \\
& =\frac{\gamma}{\theta \mathrm{B}(\alpha, \beta-1 / \gamma)}\left[1-\left(\frac{\mathrm{x}}{\theta}\right)^{-\gamma}\right]^{\alpha-1}\left(\frac{\mathrm{x}}{\theta}\right)^{-\gamma \beta}
\end{aligned}
$$


By setting $y=(x / \theta)^{-\gamma}$ in (4), it is not difficult to show that $\int_{\theta}^{\infty} \mathrm{f}_{\mathrm{L}}(\mathrm{x}) \mathrm{dx}=1$. If $\mathrm{X}$ has PDF as (4), we shall write $\mathrm{X} \sim \operatorname{LBBP}(\alpha, \beta, \theta, \gamma)$.

The CDF of LBBP r.v., denoted as $\mathrm{F}_{\mathrm{L}}(\mathrm{x})$, can be rewrite as $F_{L}(x)=1-F_{L}^{*}(x)$, then, $F_{L}^{*}(x)$ for PDF in (4) is:

$$
\mathrm{F}_{\mathrm{L}}^{*}(\mathrm{x})=\int_{\mathrm{x}}^{\infty} \frac{\gamma}{\theta \mathrm{B}(\alpha, \beta-1 / \gamma)}\left[1-\left(\frac{\mathrm{t}}{\theta}\right)^{-\gamma}\right]^{\alpha-1}\left(\frac{\mathrm{t}}{\theta}\right)^{-\gamma \beta} \mathrm{dt}, \mathrm{t} \geq \theta
$$

By setting $y=(t / \theta)^{-\gamma}, 0<y<1$, the above integration becomes:

$$
\mathrm{F}_{\mathrm{L}}^{*}(\mathrm{x})=\int_{0}^{2} \frac{1}{\mathrm{~B}(\alpha, \beta-1 / \gamma)}(1-\mathrm{y})^{\alpha-1} \mathrm{y}^{\beta-\frac{1}{\gamma}-1} \mathrm{dy}=\frac{\mathrm{B}(\mathrm{z} ; \beta-1 / \gamma, \alpha)}{\mathrm{B}(\alpha, \beta-1 / \gamma)}
$$

$\mathrm{B}(\mathrm{z} ; \beta-1 / \gamma, \alpha)$ is an incomplete beta function with $\mathrm{z}=$ $(\mathrm{x} / \theta)^{-\gamma}, 0<\mathrm{z}<1$, defined by:

$$
\begin{aligned}
\mathrm{B}(\mathrm{z} ; \beta-1 / \gamma, \alpha)=\mathrm{z}^{\beta-1 / \gamma}\{ & \frac{1}{(\beta-1 / \gamma)}+\frac{1-\alpha}{(\beta-1 / \gamma)+1} \mathrm{z} \\
& \left.+\ldots+\frac{(1-\alpha)(2-\alpha) \ldots(\mathrm{n}-\alpha)}{\mathrm{n} ![(\beta-1 / \gamma)+\mathrm{n}]} \mathrm{Z}^{\mathrm{n}}+\ldots\right\}
\end{aligned}
$$

Therefore:

$$
F_{L}(x)=1-\frac{B(z ; \beta-1 / \gamma, \alpha)}{B(\alpha, \beta-1 / \gamma)}
$$

We display the graphs of the LBBP distribution in some parameters values of $\alpha, \beta$ and $\gamma$ in Fig. 1. Due to the fact that, $\gamma$ and $\beta$ are direct variation, but $\theta$ reverse variation with behavior kurtosis in these graphs.

We consider some sub-models of the LBBP distribution in the following corollaries.

\section{Corollary 1:}

If $\alpha=\beta=1$ it is easy to show that the LBBP distribution reduces to the Length Biased Pareto (LBP) distribution (Patil and Rao, 1978) with PDF:

$$
f_{L}(x)=\frac{(\gamma-1) \theta^{\gamma-1}}{x^{\gamma}}, x \geq \theta ; \theta>0, \gamma>1
$$

\section{Corollary 2:}

If $\mathrm{X} \sim \operatorname{LBBP}(\alpha=1 / 2, \beta=(1 / 2)+(1 / \gamma), \theta, \gamma)$, then the r.v. $\mathrm{Y}=(\mathrm{X} / \theta)^{-\gamma}$, the LBBP distribution transform to arcsine distribution with PDF:

$$
f(y)=\frac{1}{\pi \sqrt{y(1-y)}}, 0<y<1
$$

\section{Proof:}

Using a transformation method, we can show that r.v. $\mathrm{Y}$ has the PDF as follows:

$$
\begin{aligned}
\mathrm{f}(\mathrm{y}) & =\frac{\gamma}{\theta \mathrm{B}\left(\frac{1}{2}, \frac{1}{2}+\frac{1}{\gamma}-\frac{1}{\gamma}\right)}(1-\mathrm{y})^{\frac{1}{2}-1} \mathrm{y}^{\frac{1}{2}+\frac{1}{\gamma}}\left|\frac{-\theta}{\gamma} \mathrm{y}^{-1-\frac{1}{\gamma}}\right| \\
& =\frac{(1-\mathrm{y})^{-\frac{1}{2}} \mathrm{y}^{-\frac{1}{2}}}{\Gamma\left(\frac{1}{2}\right) \Gamma\left(\frac{1}{2}\right)}=\frac{1}{\pi \sqrt{\mathrm{y}(1-\mathrm{y})}}
\end{aligned}
$$

\section{Corollary 3:}

If $\mathrm{X} \sim \operatorname{LBBP}(\alpha, \beta, \theta, \gamma)$, then $\mathrm{Y}=\beta \ln (\mathrm{X} / \theta)$ has the $\log$ beta distribution (Dufresne, 2007), with parameter $\alpha, \gamma / \beta$ and $\gamma-(1 / \beta)$ with PDF Equation (6):

$$
\mathrm{f}(\mathrm{y})=\frac{\gamma}{\beta \mathrm{B}(\alpha, \beta-1 / \gamma)}\left(1-\mathrm{e}^{\frac{-\gamma y}{\beta}}\right)^{\alpha-1} \mathrm{e}^{-\left(\gamma-\frac{1}{\beta}\right) y}, 0<\mathrm{y}<1
$$

\section{Proof:}

By a method of transformation, it shows that:

$$
\begin{aligned}
\mathrm{f}(\mathrm{y}) & =\frac{\gamma}{\theta \mathrm{B}(\alpha, \beta-1 / \gamma)}\left(1-\mathrm{e}^{\frac{-\gamma y}{\beta}}\right)^{\alpha-1} \mathrm{e}^{\frac{-\gamma \beta y}{\beta}}\left|\frac{\theta \mathrm{e}^{\frac{y}{\beta}}}{\beta}\right| \\
& =\frac{\gamma}{\beta \mathrm{B}(\alpha, \beta-1 / \gamma)}\left(1-\mathrm{e}^{\frac{-\gamma y}{\beta}}\right)^{\alpha-1} \mathrm{e}^{-\left(\gamma \cdot \frac{1}{\beta}\right) y}
\end{aligned}
$$

Therefore, $\mathrm{f}(\mathrm{y})$ can be obtained by setting $\mathrm{a}=\alpha, \mathrm{b}=$ $\gamma / \beta$ and $c=\gamma-(1 / \beta)$, as:

$$
f(y)=\frac{b}{B(a, c / b)}\left(1-e^{-b y}\right)^{a-1} e^{-c y}
$$




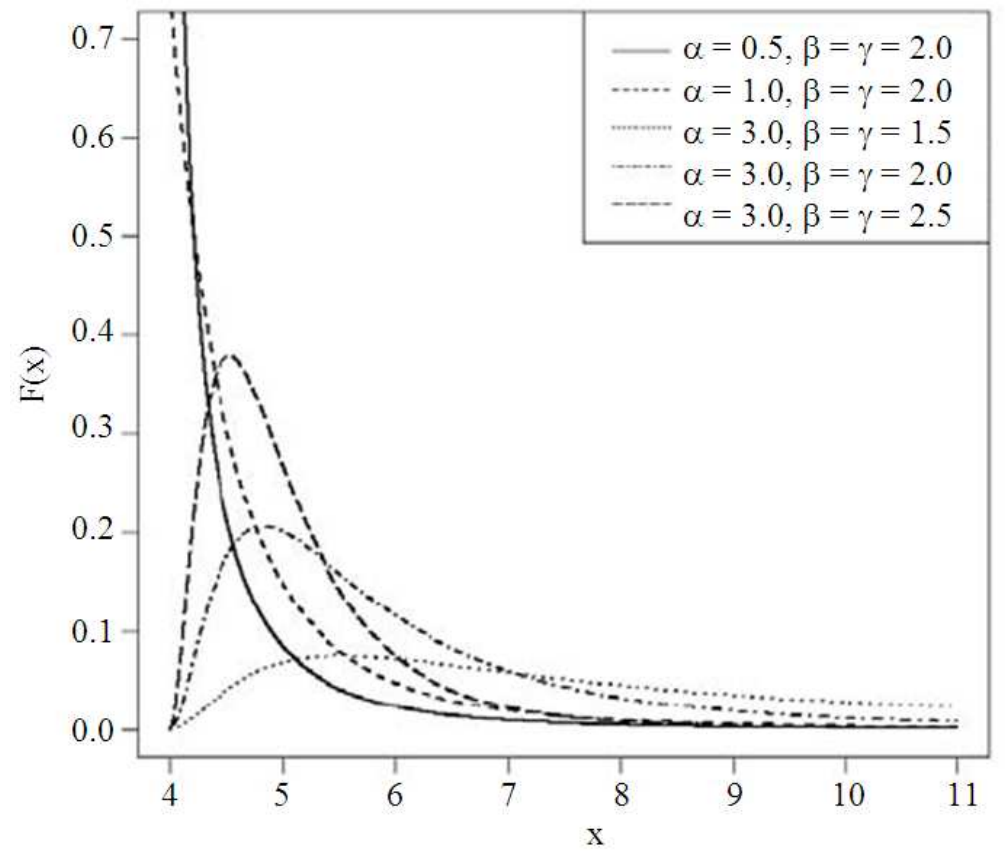

Fig. 1. The PDF of LBBP r.v. $X$ for $\theta=4$ of some values of $\alpha$ and $\beta=\gamma$

\section{Corollary 4:}

If $\mathrm{X} \sim \operatorname{LBBP}(\alpha=1, \beta, \theta, \gamma)$, then $\mathrm{Y}=\beta \ln (\mathrm{X} / \theta)$ is follows exponential distribution with mean $1 /[\gamma-(1 / \beta)]$ :

$$
f(y)=\left(\gamma-\frac{1}{\beta}\right) \mathrm{e}^{-\left(\gamma-\frac{1}{\beta}\right) y}, 0<y<1
$$

\section{Proof:}

By setting $\alpha=1$ in (6), the result follows that.

\section{Corollary 5:}

If $\mathrm{X} \sim \operatorname{LBBP}(\alpha, \beta=\mathrm{b}-(1 / \gamma), \theta, \gamma)$, then the $\operatorname{LBBP}$ distribution transfer to the BP distribution (Akinsete et al., 2008) with PDF:

$$
f(x)=\frac{\gamma}{\theta B(\alpha, b)}\left[1-\left(\frac{x}{\theta}\right)^{-\gamma}\right]^{\alpha-1}\left(\frac{x}{\theta}\right)^{-\gamma b-1}, x \geq \theta ; \alpha, b, \theta, \gamma>0
$$

\subsection{Other Types of LBBP Distribution}

Let $\mathrm{X}$ has the LBBP distribution. The PDF of LBBP type II, III and IV are, respectively, given by:

$$
\begin{aligned}
& f_{\text {II }}(x)=\frac{a x / C}{\theta B(\alpha, \beta-1 / \gamma)}\left[1-\left(1+\frac{x}{C}\right)^{-a}\right]^{\alpha-1}\left(1+\frac{x}{C}\right)^{-a \beta-1} \\
& f_{\text {III }}(x)=\frac{x[b+a /(x+C)]}{\theta B(\alpha, \beta-1 / \gamma)}\left[1-\frac{C e^{-b x}}{(x+C)^{a}}\right]^{\alpha-1}\left[\frac{C e^{-b x}}{(x+C)^{a}}\right]^{\beta}
\end{aligned}
$$

and:

$$
\begin{aligned}
\mathrm{f}_{\mathrm{IV}}(\mathrm{x})= & \frac{\mathrm{x} \xi}{\lambda \theta \sigma \mathrm{B}(\alpha, \beta-1 / \gamma)}\left[\left(\frac{\mathrm{x}-\mu}{\sigma}\right)^{1 / \lambda-1}\right] \\
& \times\left\{1-\left[1+\left(\frac{\mathrm{x}-\mu}{\sigma}\right)^{1 / \lambda}\right]^{-\xi}\right\}^{\alpha-1}\left[1+\left(\frac{\mathrm{x}-\mu}{\sigma}\right)^{1 / \lambda}\right]^{-\xi \beta-1}
\end{aligned}
$$

Since, the PDF of LBBP distribution in (4) is originated (Eugene et al., 2002):

$$
f_{L}(x)=\frac{x}{\theta B(\alpha, \beta-1 / \gamma)}[F(x)]^{\alpha-1}[1-F(x)]^{\beta-1} F^{\prime}(x)
$$

\subsection{Hazard Rate}

Hazard rate (or failure rate) and survival function are extensively apply in many fields. For instance; the 
trivariate hazard rate function of trivariate liftime distribution was presented by Wahyudi et al. (2011).

By definition, the hazard rate of $\mathrm{X}$ is given by:

$$
h(x)=\frac{f(x)}{1-F(x)}
$$

where, $\mathrm{f}(\mathrm{x})$ and $\mathrm{F}(\mathrm{x})$ are $\mathrm{PDF}$ and $\mathrm{CDF}$ of $\mathrm{X}$, respectively. Using (4) and (5), the hazard rate of the LBBP distribution may be expressed as:

$$
\begin{aligned}
\mathrm{h}(\mathrm{x})= & \frac{\gamma}{\theta \mathrm{B}(\mathrm{z} ; \beta-1 / \gamma, \alpha)}\left[1-\left(\frac{\mathrm{x}}{\theta}\right)^{-\gamma}\right]^{\alpha-1}\left(\frac{\mathrm{x}}{\theta}\right)^{-\gamma \beta} \\
\mathrm{h}(\mathrm{x})= & \frac{\frac{\gamma}{\mathrm{x}}\left[1-\left(\frac{\mathrm{x}}{\theta}\right)^{-\gamma}\right]^{\alpha-1}}{\left[\frac{1}{\beta-1 / \gamma}+\frac{1-\alpha}{\beta+1-1 / \gamma}\left(\frac{\mathrm{x}}{\theta}\right)^{-\gamma}+\ldots\right.} \\
& \left.+\frac{(1-\alpha)(2-\alpha) \ldots(\mathrm{n}-\alpha)}{\mathrm{n} !(\beta+\mathrm{n}-1 / \gamma)}\left(\frac{\mathrm{x}}{\theta}\right)^{-\gamma \mathrm{n}}+\ldots\right]
\end{aligned}
$$

We display some graphs of hazard rate for the LBBP distribution in Fig. 2. It is noted that by setting $\alpha=\beta=1$ in $h(x)$, we have the hazard rate of the LBP distribution.

\subsection{Moments of LBBP Distribution}

The result of this section gives the rth moments of the LBBP distribution. Some of the most important mathematical properties of distribution can be studied through rth moments.

\section{Definition 2:}

Let $\mathrm{X}$ be a nonnegative r.v. with the rth moments, $E\left(X^{r}\right)<\infty$. Then the $E_{L}\left(X^{r}\right)$ for length biased distribution of $\mathrm{X}$ can be obtained by:

$$
E_{L}\left(X^{r}\right)=\frac{E\left(X^{r+1}\right)}{E(X)}, r=1,2,3, \ldots
$$

\section{Theorem 2:}

Let $\mathrm{X} \sim \operatorname{LBBP}(\alpha, \beta, \theta, \gamma)$. The $r$ th moments of $\mathrm{X}$ is given by Equation (7):

$$
\begin{aligned}
& \mathrm{E}_{\mathrm{L}}\left(\mathrm{X}^{\mathrm{r}}\right)=\theta^{\mathrm{r}} \frac{\Gamma(\beta-(\mathrm{r}+1) / \gamma) \Gamma(\alpha+\beta-1 / \gamma)}{\Gamma(\alpha+\beta-(\mathrm{r}+1) / \gamma) \Gamma(\beta-1 / \gamma)}, \\
& \alpha, \beta, \theta, \gamma>0 ; r=1,2,3, \ldots
\end{aligned}
$$

\section{Proof:}

If $\mathrm{X} \sim \operatorname{LBBP}(\alpha, \beta, \theta, \gamma)$, it is easy to find the $\mathrm{rth}$ moments of X, By replacing (2) and (3) in Definition 2, then $E_{L}\left(X^{r}\right)$ is obtained as follows:

$$
\begin{aligned}
\mathrm{E}_{\mathrm{L}}\left(\mathrm{X}^{\mathrm{r}}\right) & =\theta^{\mathrm{r}} \frac{\mathrm{B}(\alpha, \beta-(\mathrm{r}+1) / \gamma)}{\mathrm{B}(\alpha, \beta-1 / \gamma)} \\
& =\theta^{\mathrm{r}} \frac{\Gamma(\beta-(\mathrm{r}+1) / \gamma) \Gamma(\alpha+\beta-1 / \gamma)}{\Gamma(\alpha+\beta-(\mathrm{r}+1) / \gamma) \Gamma(\beta-1 / \gamma)}
\end{aligned}
$$

From (7), it is simple to deduce mean and variance of $\mathrm{X}$ which are given in (8) and (9) respectively Equation (8 and 9):

$$
\begin{aligned}
\mathrm{E}_{\mathrm{L}}(\mathrm{X})=\theta & \frac{\Gamma(\beta-2 / \gamma) \Gamma(\alpha+\beta-1 / \gamma)}{\Gamma(\alpha+\beta-2 / \gamma) \Gamma(\beta-1 / \gamma)} \\
\operatorname{Var}(\mathrm{X})=\theta^{2} & \left\{\left[\frac{\Gamma(\beta-3 / \gamma) \Gamma(\alpha+\beta-1 / \gamma)}{\Gamma(\alpha+\beta-3 / \gamma) \Gamma(\beta-1 / \gamma)}\right]\right. \\
& \left.-\left[\frac{\Gamma(\beta-2 / \gamma) \Gamma(\alpha+\beta-1 / \gamma)}{\Gamma(\alpha+\beta-2 / \gamma) \Gamma(\beta-1 / \gamma)}\right]^{2}\right\}
\end{aligned}
$$

We let $\omega(\alpha, \beta, \gamma ; \mathrm{i})=\frac{\Gamma(\beta-\mathrm{i} / \gamma) \Gamma(\alpha+\beta-1 / \gamma)}{\Gamma(\alpha+\beta-\mathrm{i} / \gamma) \Gamma(\beta-1 / \gamma)}, \beta>\mathrm{i} / \gamma, \mathrm{i} \in \mathrm{I}^{+}$ and $\mathrm{T}=\sqrt{\omega(\alpha, \beta, \gamma ; 3)-\omega^{2}(\alpha, \beta, \gamma ; 2)}$. So, the skewness and kurtosis are independent from parameter $\theta$ as follows Equation (10 and 11):

$$
\begin{aligned}
& \operatorname{Skewness}(\mathrm{X})=\mathrm{T}^{-3}\left\{\omega(\alpha, \beta, \gamma ; 4)-2 \omega^{3}(\alpha, \beta, \gamma ; 2)\right. \\
&-3 \omega(\alpha, \beta, \gamma ; 3) \omega(\alpha, \beta, \gamma ; 2)\} \\
& \operatorname{Kurtosis}(\mathrm{X})=\mathrm{T}^{-4}\left\{\omega(\alpha, \beta, \gamma ; 5)-3 \omega^{4}(\alpha, \beta, \gamma ; 2)\right. \\
&-4 \omega(\alpha, \beta, \gamma ; 4) \omega(\alpha, \beta, \gamma ; 2) \\
&\left.+6 \omega(\alpha, \beta, \gamma ; 3) \omega^{2}(\alpha, \beta, \gamma ; 2)\right\}
\end{aligned}
$$

We furnish Table 1 which contains the mean in (8), variance in (9), skewness in (10) and kurtosis in (11) for diverse values of parameters. From Table 1, we examine the mean is closed to $\theta$, the mean and variance are increasing functions of $\alpha$ and $\theta$, but are decreasing functions of $\beta$ and $\gamma$. Moreover, the skewness and kurtosis are decreasing functions of $\alpha, \beta$ and $\gamma$. 


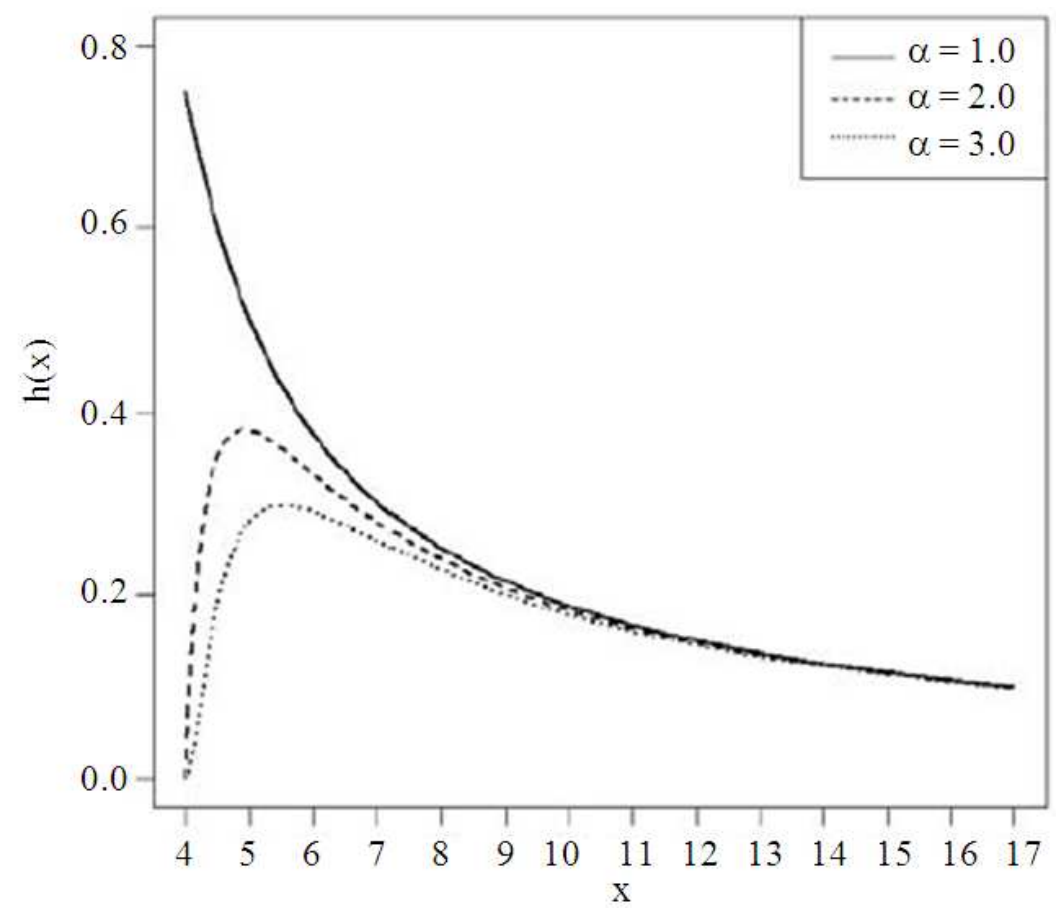

Fig. 2. The hazard rates of LBBP r.v. X for $\beta=\gamma=2, \theta=4$ and some values of $\alpha$

Table 1. Mean, variance, skewness and kurtosis of the LBBP distribution for different values of $\alpha, \beta, \gamma$ and $\theta$

\begin{tabular}{|c|c|c|c|c|c|c|c|}
\hline$\beta$ & $\alpha$ & $\gamma$ & $\theta$ & Mean & Variance & Skewness & Kurtosis \\
\hline \multirow[t]{8}{*}{2.0} & \multirow[t]{4}{*}{0.75} & \multirow[t]{2}{*}{5.0} & 2.0 & 2.1973 & 0.0647 & \multirow[t]{2}{*}{3.2558} & \multirow[t]{2}{*}{23.2135} \\
\hline & & & 5.0 & 5.4932 & 0.4043 & & \\
\hline & & \multirow[t]{2}{*}{10.0} & 2.0 & 2.0879 & 0.0113 & \multirow[t]{2}{*}{2.6575} & \multirow[t]{2}{*}{14.6414} \\
\hline & & & 5.0 & 5.2197 & 0.0705 & & \\
\hline & \multirow{4}{*}{5.0} & \multirow[t]{2}{*}{5.0} & 2.0 & 2.7642 & 0.2165 & \multirow[t]{2}{*}{2.0084} & \multirow[t]{2}{*}{11.6366} \\
\hline & & & 5.0 & 6.9105 & 1.3532 & & \\
\hline & & \multirow[t]{2}{*}{10.0} & 2.0 & 2.3303 & 0.0315 & \multirow[t]{2}{*}{1.4673} & \multirow[t]{2}{*}{7.0239} \\
\hline & & & 5.0 & 5.8257 & 0.1971 & & \\
\hline \multirow[t]{8}{*}{7.5} & \multirow[t]{4}{*}{0.75} & \multirow[t]{2}{*}{5.0} & 2.0 & 2.0428 & 0.0026 & \multirow[t]{2}{*}{2.4881} & \multirow[t]{2}{*}{12.7552} \\
\hline & & & 5.0 & 5.1071 & 0.0161 & & \\
\hline & & \multirow[t]{2}{*}{10.0} & 2.0 & 2.0209 & 0.0006 & \multirow[t]{2}{*}{2.3931} & \multirow[t]{2}{*}{11.7955} \\
\hline & & & 5.0 & 5.0521 & 0.0037 & & \\
\hline & \multirow[t]{4}{*}{5.0} & \multirow[t]{2}{*}{5.0} & 2.0 & 2.2356 & 0.0130 & \multirow[t]{2}{*}{1.1224} & \multirow[t]{2}{*}{5.1367} \\
\hline & & & 5.0 & 5.5891 & 0.0814 & & \\
\hline & & \multirow[t]{2}{*}{10.0} & 2.0 & 2.1126 & 0.0028 & \multirow[t]{2}{*}{1.0196} & \multirow[t]{2}{*}{4.6838} \\
\hline & & & 5.0 & 5.2814 & 0.0173 & & \\
\hline
\end{tabular}

\subsection{Rényi and Shannon Entropies}

Statistical entropy is a probabilistic measure of ignorance about the outcome of a random experiment and is a measure of a reduction in that uncertainty. Entropy of $X$ with PDF $f(x)$ is a measure of variation of the uncertainty (Nadarajah and Kotz, 2006).
Rényi entropy is defined by Equation (12):

$$
I_{R}(\xi)=\frac{1}{1-\xi} \log \left[\int f^{\xi}(x) d x\right]
$$

where, $\xi>0$ and $\xi \neq 1$ for the PDF of LBBP distribution in (4) is given by: 
$\int_{\theta}^{\infty} f_{L}^{\xi}(x) d x=\frac{\gamma^{\xi}}{[\theta B(\alpha, \beta-1 / \gamma)]^{\xi}} \int_{\theta}^{\infty}\left[1-\left(\frac{x}{\theta}\right)^{-\gamma}\right]^{\xi(\alpha-1)}\left(\frac{x}{\theta}\right)^{-\xi \gamma \beta} d x$

Substituting $y=1-(x / \theta)^{-\gamma}$, it is not intricate to show that:

$$
\int_{\theta}^{\infty} f_{L}^{\xi}(x) d x=\left(\frac{\gamma}{\theta}\right)^{\xi-1} \frac{B(\xi(\alpha-1)+1, \xi \beta-1 / \gamma)}{B^{\xi}(\alpha, \beta-1 / \gamma)}
$$

The Rényi entropy can be written as Equation (13):

$\mathrm{I}_{\mathrm{R}}(\xi)=\log \left(\frac{\theta}{\gamma}\right)+\frac{1}{1-\xi} \log \left[\frac{\mathrm{B}(\xi(\alpha-1)+1, \xi \beta-1 / \gamma)}{\mathrm{B}^{\xi}(\alpha, \beta-1 / \gamma)}\right]$

Shannon entropy can be obtained as $E[-\log f(X)]$. It is the special case of (12) for $\xi \rightarrow 1$, is given by:

$$
E[-\log f(X)]=\lim _{\xi \rightarrow 1} I_{R}(\xi)
$$

Limiting $\xi \rightarrow 1$ in (13) and using L'Hospital's rule, the Shannon entropy can be expanded as:

$$
\begin{aligned}
E\left[-\log f_{L}(X)\right]= & \log \left(\frac{\theta}{\gamma}\right)-(\alpha-1) \Psi(\alpha)-\beta \Psi(\beta-1 / \gamma) \\
& +(\alpha-1+\beta) \Psi(\alpha+\beta-1 / \gamma)+\log B(\alpha, \beta-1 / \gamma)
\end{aligned}
$$

where, $\Psi(\mathrm{z})=\Gamma^{\prime}(\mathrm{z}) / \Gamma(\mathrm{z})$ is a digamma function.

\section{RESULTS}

\subsection{Parameters Estimation}

The estimation of parameters for the LBBP distribution via the MLE will be discussed. Let $X_{1}, \ldots, X_{n}$ be a random sample from $\mathrm{X} \sim \operatorname{LBBP}(\alpha, \beta, \theta, \gamma)$ the likelihood function is given by:

$$
\mathrm{L}(\mathrm{x} ; \alpha, \beta, \theta, \gamma)=\prod_{j=1}^{\mathrm{n}}\left\{\frac{\gamma}{\theta \mathrm{B}(\alpha, \beta-1 / \gamma)}\left[1-\left(\frac{\mathrm{x}_{\mathrm{j}}}{\theta}\right)^{-\gamma}\right]^{\alpha-1}\left(\frac{\mathrm{x}_{\mathrm{j}}}{\theta}\right)^{-\gamma \beta}\right\}
$$

With corresponding log-likelihood function Equation (14):

$$
\begin{aligned}
\operatorname{lnL}(\alpha, \beta, \theta, \gamma)= & \mathrm{n} 1 \mathrm{n} \gamma-\mathrm{n} 1 \mathrm{n} \theta-\mathrm{n} 1 \mathrm{n} \Gamma(\alpha)-\mathrm{n} 1 \mathrm{n} \Gamma(\beta-1 / \gamma) \\
& +\mathrm{n} 1 \mathrm{n} \Gamma(\alpha+\beta-1 / \gamma)-\gamma \beta \sum_{\mathrm{j}=1}^{\mathrm{n}} 1 \mathrm{n}\left(\frac{\mathrm{x}_{\mathrm{j}}}{\theta}\right) \\
& +(\alpha-1) \sum_{\mathrm{j}=1}^{\mathrm{n}} 1 \mathrm{n}\left[1-\left(\frac{\mathrm{x}_{\mathrm{j}}}{\theta}\right)^{-\gamma}\right]
\end{aligned}
$$

The maximum likelihood estimate of $\hat{\theta}$ is the firstorder statistic $\mathrm{x}_{(1)}$. The first order conditions for finding the optimal values of the parameters obtained by differentiating in (14) with respect to $\alpha, \beta$ and $\gamma$ give rise to the following differential Equation (15 and 16):

$$
\begin{aligned}
\frac{\partial}{\partial \alpha} \ln L(x)= & n[\Psi(\alpha+\beta-1 / \gamma)-\Psi(\alpha)] \\
& +\sum_{j=1}^{n} \ln \left[1-\left(\frac{x_{j}}{\theta}\right)^{-\gamma}\right] \\
\frac{\partial}{\partial \beta} \ln L(x)= & n[\Psi(\alpha+\beta-1 / \gamma)-\Psi(\beta-1 / \gamma)] \\
& -\gamma \sum_{j=1}^{n} \ln \left(\frac{x_{j}}{\theta}\right)
\end{aligned}
$$

and Equation (17):

$$
\begin{aligned}
\frac{\partial}{\partial \gamma} \operatorname{lnL}(\mathrm{x})= & \frac{\mathrm{n}}{\gamma}+\mathrm{n}[\Psi(\alpha+\beta-1 / \gamma)-\Psi(\beta-1 / \gamma)]-\sum_{\mathrm{j}=1}^{\mathrm{n}} \\
& \left\{\beta+(\alpha-1)\left[1-\left(\frac{\mathrm{x}_{\mathrm{j}}}{\theta}\right)^{\gamma}\right]^{-1}\right\} \ln \left(\frac{\mathrm{x}_{\mathrm{j}}}{\theta}\right)
\end{aligned}
$$

The maximum likelihood estimates $\hat{\alpha}, \hat{\beta}$ and $\hat{\gamma}$, respectively, are taken by solving iteratively (15)-(17) to zero. These differential equations are not in close form, a numerical method can be employed to obtain the expectations of them.

\subsection{Application of LBBP Distribution}

In this section, the LBBP distribution is fitted to a real data set, we consider the data set in the field of insurance which has received extensive attention in the actuarial literature. This data set is one among the twenty sets of Norwegian fire claims (in millions of Norwegian krones) is presented in Fernandez (2013). The parameter estimation for Norwegian fire claims data and K$\mathrm{S}$ statistics are shown in Table 2. In this case, since the values of the K-S statistics are smaller for the LBBP distribution compared to those values of the LBP and BP distributions. 
Table 2. Parameter estimates and K-S statistics for Norwegian fire claims data

\begin{tabular}{llll}
\hline Distribution & LBP & BP & LBBP \\
\hline Parameter & $\hat{\theta}=0.5$ & $\hat{\theta}=0.5$ & $\hat{\theta}=0.5$ \\
estimates & $\hat{\gamma}=2.2175$ & $\hat{\gamma}=169.1104$ & $\hat{\gamma}=113.9502$ \\
& & $\hat{\alpha}=0.3183$ & $\hat{\alpha}=0.3703$ \\
& & $\hat{\beta}=0.0069$ & $\hat{\beta}=0.0190$ \\
K-S statistics & 0.0505 & 0.0444 & 0.0425 \\
p-value & 0.862 & 0.942 & 0.959 \\
\hline
\end{tabular}

\section{DISCUSSION}

The LBBP distribution is consequence of length biased distribution method which is a new generalized of Pareto distribution. In this study, the LBBP distribution found that it provides a significantly better fit than the LBP and BP distributions which are some sub-models of the LBBP distribution. As well as the research of Mahmoudi (2011) found the beta generalized Pareto distribution using the exceedances of flood peaks of the Wheaton River near Carcross in Yukon Territory, Canada provides a better fit than sub-models of it. Furthermore, the result of this study consistent with the findings of Das and Roy (2011), the length biased Weibull distribution provided fit to data of June rainfall in Tezpur Assam, India better than its sub-models.

\section{CONCLUSION}

We proposed the Length Biased Beta-Pareto (LBBP) distribution. We found some well-known sub-models such as; LBP, arcsine, log-beta, exponential, BP distributions and LBBP type II, III and IV. Rényi and Shannon entropies and hazard rate are provided. We derive the rth moments and apply MLE to estimate parameters of the distribution. An application to a real data set shows that the fit of the LBBP distribution is best fit to the data with highest p-value. We hope the LBBP distribution may attract extensive applications in lifetime data analysis and other fields. The future research may consider in parameter estimation using Bayesian or other approaches. In addition, a new mixture between the LBBP and BP distributions will be developed.

\section{ACKNOWLEDGEMENT}

This research was made possible by Faculty of Science, Kasetsart University and National Statistics Office, Thailand. We thank Dr. Chookait Pudprommarat and anonymous reviewers for comments made on an earlier version of this manuscript and for many inspiring suggestions.

\section{REFERENCES}

Ahmed, A., K.A. Mir and J.A. Reshi, 2013. On new method of estimation of parameters of size-biased generalized gamma distribution and its structural properties. IOSR J. Math., 5: 34-40.

Akinsete, A., F. Famoye and C. Lee, 2008. The betaPareto distribution. Statistics, 42: 547-563. DOI: 10.1080/02331880801983876

Ali, M.M. and S. Nadarajah, 2006. A truncated Pareto distribution. Comput. Commun., 30: 1-4. DOI: 10.1016/j.comcom.2006.07.003

Alkarni, S.H., 2012. New family of logarithmic lifetime distributions. J. Math. Stat., 8: 435-440. DOI: 10.3844/jmssp.2012.435.440

Das, K.K. and T.D. Roy, 2011. On some length-biased weighted Weibull distribution. Adv. Applied Sci. Res., 2: 465-475.

Dufresne, D., 2007. Fitting combinations of exponentials to probability distributions. Applied Stochastic Models Bus. Ind., 23: 23-48. DOI: 10.1002/asmb.635

Eugene, N., C. Lee and F. Famoye, 2002. The betanormal distribution and its applications. Commun. Statist.-Theory Meth., 31: 497-512. DOI: 10.1081/STA-120003130

Fernandez, A.J., 2013. Smallest Pareto confidence regions and applications. Comput. Statist. Data Anal., 62: 11-25. DOI: 10.1016/j.csda.2012.12.016

Johnson, N.L., S. Kotz and N. Balakrishnan, 1995. Continuous Univariate Distributions. 2nd Edn., John Wiley and Sons, New York, ISBN-10: 0471584940, pp: 752.

Khattree, R., 1989. Characterization of inverse-Gaussian and gamma distributions through their length-biased distributions. IEEE Trans. Reliab., 38: 610-611. DOI: $10.1109 / 24.46490$

Lawless, J.F., 2011. Statistical Models and Methods for Lifetime Data. 2nd Edn., John Wiley and Sons, Hoboken, N.J., ISBN-10: 1118031253, pp: 664.

Mahmoudi, E., 2011. The beta generalized Pareto distribution with application to lifetime data. Math. Comput. Simulat., 81: 2414-2430. DOI: 10.1016/j.matcom.2011.03.006

Manas, A., 2011. The paretian ratio distribution-an application to the volatility of GDP. Econ. Lett., 111: 180-183. DOI: 10.1016/j.econlet.2011.01.026 
Nadarajah, S. and S. Kotz, 2006. The beta exponential distribution. Reliab. Eng. Syst. Safety, 91: 689-697. DOI: 10.1016/j.ress.2005.05.008

Nadarajah, S., 2005. Exponentiated Pareto distributions. Statistics, 39: 255-260. DOI: 10.1080/02331880500065488

Nassar, M. and N. Nada, 2013. A new generalization of the Pareto-geometric distribution. J. Egy. Math. Soc., 21: 148-155. 10.1016/j.joems.2013.01.003

Newman, M.E.J., 2005. Power laws, Pareto distributions and Zipf's law. Contemporary Phy., 46: 323-351. DOI: $10.1080 / 00107510500052444$

Oluyede, B.O. and E.O. George, 2002. On stochastic inequalities and comparisons of reliability measures for weighted distributions. Math. Prob. Eng., 8: 113. DOI: $10.1080 / 10241230211380$
Oluyede, B.O., 2006. A note on probability weighted moment inequalities for reliability measures. J. Inequal. Pure Applied Math.

Patil, G.P. and C.R. Rao, 1978. Weighted distributions and size-biased sampling with applications to wildlife populations and human families. Biometrics, 34: 179-189.

Rao, C.R., 1965. On discrete distributions arising out of methods of ascertainment. Sankhyā: Indian J. Statist., Series A., 27: 311-324.

Wahyudi, I., Purhadi, Irhamah and Sutikno, 2011. The development of parameter estimation on hazard rate of trivariate weibull distribution. Am. J. Biostat., 2: 26-35. DOI: 10.3844/amjbsp.2011.26.35 